\title{
Characteristics of Crystallization of Complex Plasmas in Narrow Channels
}

\author{
B. A. Klumov and G. E. Morfill \\ Max-Planck-Institut für Extraterrestrische Physik, D-85740 Garching, Germany \\ e-mail:klumov@mpe.pmg.de \\ Received April 25, 2008
}

\begin{abstract}
Molecular dynamics simulations are performed to analyze the dependence of the behavior of complex (dusty) plasmas in narrow three-dimensional channels on the confining potential. Dynamics of micrometer-sized particles is modeled by using Langevin thermostat and Yukawa (screened Coulomb) pair interaction potential. A detailed analysis shows that confinement strongly affects plasma crystallization characteristics and local ordering of dust grains. In particular, the formation of a new, quasi-crystalline phase induced by hard-wall confinement is revealed. Transitions between different lattice symmetries induced by changes in channel width are examined. Strong dependence of the transverse dust density profile on the shielding parameter (ratio between mean interparticle distance and screening length) can be used to manipulate the dust-grain flux in such a system.
\end{abstract}

PACS numbers: 52.27.Lw, 61.20.Ja, 64.60.Cn

DOI: $10.1134 / \mathrm{S} 106377610811023 \mathrm{X}$

Recent years have seen growing interest in processes taking place in plasmas containing micrometersized particles. They are generally known as complex or dusty plasmas (e.g., see [1, 2]). On the one hand, complex plasmas are found in a variety of natural systems, such as interstellar clouds, planetary rings [3], comet atmospheres [4], ionospheres and magnetospheres of planets, where the presence of dust grains frequently determines the system's state. For example, noctilucent clouds, which are located in the dusty cold upper atmosphere of the Earth [5-7], are dust structures determining ionization in the mesosphere. The presence of comet dust in the Jovian atmosphere probably [8] caused the brightening of the Jovian radiation belts during and after the collision of Comet ShoemakerLevy 9 in July of 1994 [9].

On the other hand, observation of the behavior of each individual particle provides a basis for characterizing an ensemble of dust grains on the most detailed (kinetic) level. This makes dusty plasmas an attractive tool for investigating various basic problems in physics, such as phase transitions $[10,11]$, hydrodynamic instabilities [12], or crystallization waves [13, 14].

One important problem is the behavior of charged micrometer- and nanometer-sized particles in narrow channels, where the mean interparticle distance is comparable to the channel width and the walls have an extremely strong effect on the particles. The behavior of a confined ensemble of interacting particles where one of the system's length scales is comparable to the mean interparticle distance has recently become the subject of extensive studies $[15,16]$ because of its importance for numerous applications in physics of nano- and microliquids, colloidal microparticles, capillary phenomena in nanopores [17], confinementinduced phase transitions, granular systems [18], etc. Complex plasmas are a convenient tool for examining such phenomena $[19,20]$.

In recent years, considerable progress has been made in understanding the behavior of colloidal particles in narrow channels (e.g., see [21, 22]). Many experimental results have been explained in the framework of the hard-sphere model. However, complex plasmas are different from colloidal plasmas in that the mean interparticle distance $\Delta$ is much greater than the particle size $a$. Since $\Delta \approx 100 \mu \mathrm{m}$ and $a \approx 1 \mu \mathrm{m}$ in typical experiments on complex plasmas, the behavior of confined complex plasmas has unique characteristics.

A laboratory dusty plasma is usually created by adding micrometer-sized particles to a weakly ionized plasma of a low-pressure inert gas discharge. Surface electron-ion recombination on dust grains leads to grain charging. The charge of a grain depends on its size and plasma parameters. For example, in a typical $\mathrm{RF}$ argon discharge, a micrometer-sized dust grain is charged to $Z_{\mathrm{d}} / e \sim 10^{3}$, where $e$ is the electron charge. High grain charge frequently leads to strong nonideality of the dust component: it can behave as a gas, a liquid, or a crystal. The crystalline state of the dust component of a complex plasma, known as plasma crystal, was discovered in $1994[23,24]$ after it had been theoretically predicted in 1986 [25].

The positive column of a gas discharge is a potential well where negatively charged dust grains are confined. 


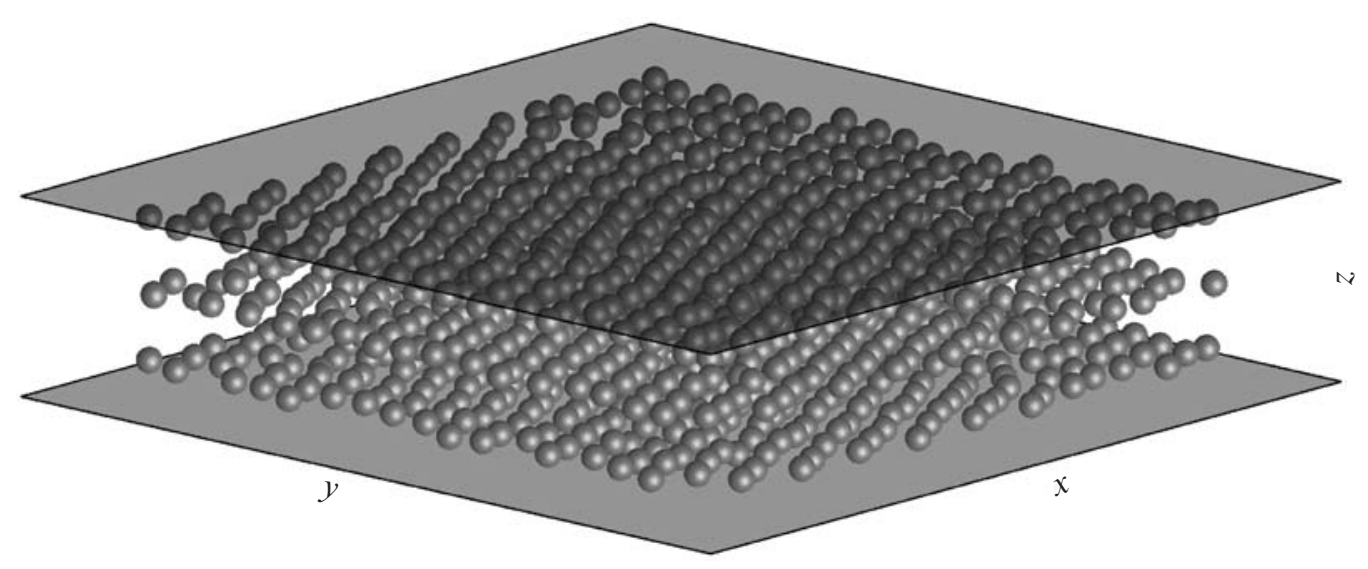

Fig. 1. Geometry of the problem. Initially, $N$ dust grains with charge $Z_{\mathrm{d}}$ are randomly distributed over the volume between two plane boundaries, which confine them to the region $0 \leq z \leq L_{z}$. In the $x y$ plane, the grains occupy the region $0 \leq x, y \leq L_{x, y}$.

In the central region, the confining potential $\Phi_{\mathrm{c}}$ can be approximated by a parabola: $\Phi_{\mathrm{c}}(x) \propto\left(x-x_{\mathrm{c}}\right)^{2}$, where $x_{\mathrm{c}}$ is the coordinate of the discharge center. Near the electrodes, the electric field gradient is much steeper and a nearly hard-wall confinement is observed. In a complex plasma experiment, the dust component can be confined by an external or self-consistent field. In the latter case, the field depends on the distribution of charged dust grains over the discharge volume.

In [19], we examined the effect of confinement on equilibrium configurations of the dust component of complex plasmas in two-dimensional narrow channels, using a screened Coulomb potential to describe the pair interactions between dust grains. In particular, it was shown that confinement strongly affects local ordering of dust grains. For example, hard-wall confinement induces transition to a glassy state in cooled dusty plasmas because of higher dust concentration at the wall, while a plasma crystal is observed in a parabolic confining potential. Local ordering and formation of layers of dust grains were observed in the experiments reported in [26].

This study focuses on local ordering of dust grains in three-dimensional narrow channels with parabolic and hard-wall confining potentials. As in two-dimensional geometry, the pair interaction between dust grains is described by a screened Coulomb (DebyeHückel or Yukawa) potential

$$
\phi(r)=\left(Z_{\mathrm{d}} / r\right) \exp \left(-r / \lambda_{\mathrm{D}}\right),
$$

where $r$ is the interparticle distance and $\lambda_{\mathrm{D}}$ is the screening length. Since we examine crystallization characteristics of the dust subsystem, we consider a strongly coupled state of a complex plasma, when the nonideality parameter of the dust subsystem is

$$
\Gamma=Z_{\mathrm{d}}^{2} \exp (-\kappa) / T_{\mathrm{d}} \Delta \gg 1,
$$

where $T_{\mathrm{d}}$ is the dust temperature and $\kappa=\Delta / \lambda_{\mathrm{D}}$ is called shielding parameter.
Behavior of an ensemble of dust grains is investigated by 3D molecular dynamics simulation. For simplicity, all dust grains are assumed to have a constant charge $Z_{\mathrm{d}}$. The dynamics of each dust grain is described by the equation of motion

$$
m \ddot{\mathbf{r}}_{i}=-Z_{\mathrm{d}} \nabla \Phi_{\mathrm{c}}-Z_{\mathrm{d}} \sum \nabla \phi-m \gamma \dot{\mathbf{r}}_{i}+\mathbf{L}_{i}
$$

The terms on the right-hand side of (1) represent the electrostatic interaction between dust grains, the drag force on the grain due to collisions with neutral atoms and buffer gas molecules, and the stochastic Langevin force $\mathbf{L}_{i}$ with autocorrelation function

$$
\left\langle\mathbf{L}_{i}(t) \mathbf{L}_{j}(t+\tau)\right\rangle=2 \gamma m k_{\mathrm{B}} \delta_{i j} \delta(\tau)
$$

and zero mean value $\left\langle\mathbf{L}_{i}(t)\right\rangle=0$, and the effect of the confining potential $\Phi_{\mathrm{c}}$.

System (1) was computed for an ensemble of $N=$ 16000 dust grains randomly distributed over the channel volume at the starting instant. Figure 1 illustrates the problem geometry. The potential $\Phi_{\mathrm{c}}$ confines the dust distribution along the $z$ axis to the interval $0 \leq z \leq$ $L_{z}$. In the $x y$ plane, the grains occupy the region $0 \leq x$, $y \leq L_{x, y}$. Periodic boundary conditions are used at $x=$ $\left\{0, L_{x}\right\}$ and $y=\left\{0, L_{y}\right\}$. The confining potential is either a parabolic one, $\Phi_{\mathrm{c}}(z) \propto\left(z-L_{z} / 2\right)^{2}$, or an elastic hard wall $\left(\Phi_{\mathrm{c}}(z) \propto \exp \left(\left(z-L_{z}\right) / \Delta_{\mathrm{w}}\right)\right.$ at $z>L_{z}$ and $\Phi_{\mathrm{c}}(z) \propto$ $\exp \left(-z / \Delta_{\mathrm{w}}\right)$ at $z<0$, where the length scale characterizes the stiffness of the wall; $\Delta_{\mathrm{w}} \approx \Delta / 3$ is used in the present simulations).

In the thermodynamic limit, the state of an infinite system of particles with Yukawa pair interaction is determined by the nonideality parameter $\Gamma$ and the shielding parameter $\kappa$ : it can crystallize into either facecentered cubic (fcc) or body-centered cubic (bcc) lattice, depending on whether $\kappa$ is large or small, respectively (e.g., see [2]). Nonequilibrium systems of Yukawa particles frequently organize into long-lived metastable phases with hexagonal close-packed (hcp) lattices (e.g., see, $[14,20]$ ), because the Gibbs free 

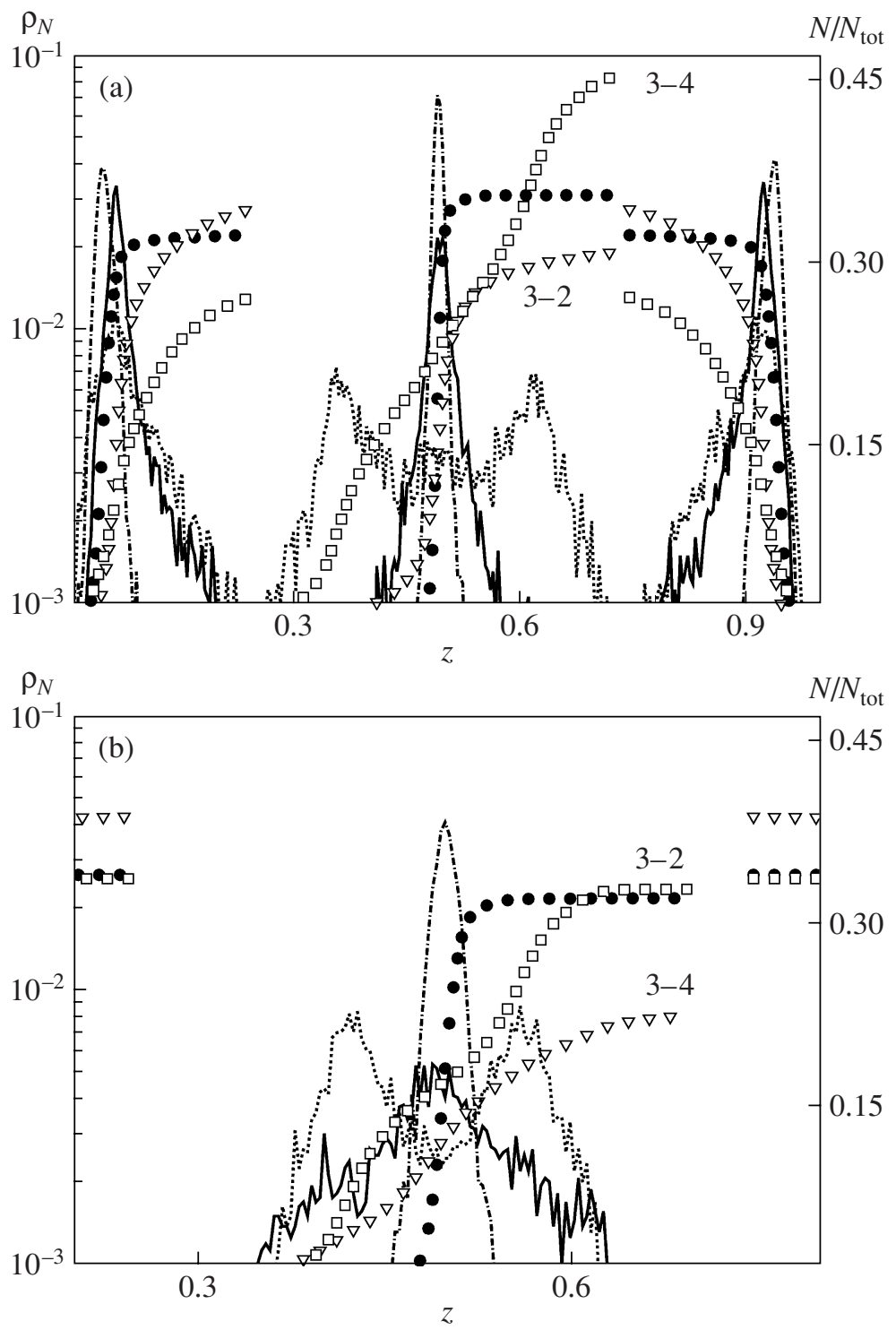

Fig. 2. Normalized dust particle density $\rho_{N}$ vs. $\tilde{z} \equiv z / L_{z}$ in (a) parabolic and (b) hard-wall confinements for several values of $L_{z}$ (i.e., $\kappa)$. In case (b), the dust distributions at the boundaries (not shown) correspond to narrow, sharply bounded symmetric layers. The values of $L_{z}$ are chosen to demonstrate the state of a dust-grain system near the bifurcation points corresponding to transformations into four-layer, two-layer, and intermediate stable three-layer structures (3-4 transition, dotted curves; 3-2 transition, solid curves; and dot-and-dash curves, respectively). Also shown are the normalized grain number $N / N_{\text {tot }}$ in each layer and $N / N_{\text {tot }}(\tilde{z})$ for $3-4$ transitions $(\square)$, 3-2 transitions $(\nabla)$, and stable three-layer system $(\bullet)$.

energies of formation of hcp and bcc phases are nearly equal [27]. To identify an fcc, bcc, or hep lattice, it is sufficient to know the ordering $A B C$ in three consecutive lattice planes. If they are known, the lattice type can be identified by visual inspection. For this reason, the behavior of a three-layer Yukawa system is examined here as an illustrative example. Some results obtained by simulating the behavior of a Yukawa system in narrow channels are presented below for confinements of different types. The parameters of the simulated Yukawa system are as follows: grain size $a \approx 1 \mu \mathrm{m}$, grain charge $Z_{\mathrm{d}} / e \approx 3 \times 10^{3}, \kappa$ varying between 2 and 3 , and neutral-gas density $\rho_{\mathrm{g}} \sim 10^{-7} \mathrm{~g} / \mathrm{cm}^{3}$.
Figure 2 shows the normalized dust density distribution $\rho_{N}$ and the normalized number of grains $N / N_{\text {tot }}$ in the three layers as functions of the distance between the layers for both types of confinement. As the system contracts (parameter $\kappa \equiv \Delta / \lambda$ decreases), the dust density goes through a bifurcation, and transition to a twolayer structure occurs at a certain point. Conversely, expansion of the system ( $\kappa$ increases), leads to the formation of a four-layer structure. Thus, the three-layer system with particular $\Gamma$ and $\lambda_{D}$ can exist only within a certain range of $\kappa$ values (determined by $L_{z}$ ).

Note also that the transverse dust density (along the $z$ axis) changes significantly as the system approaches 

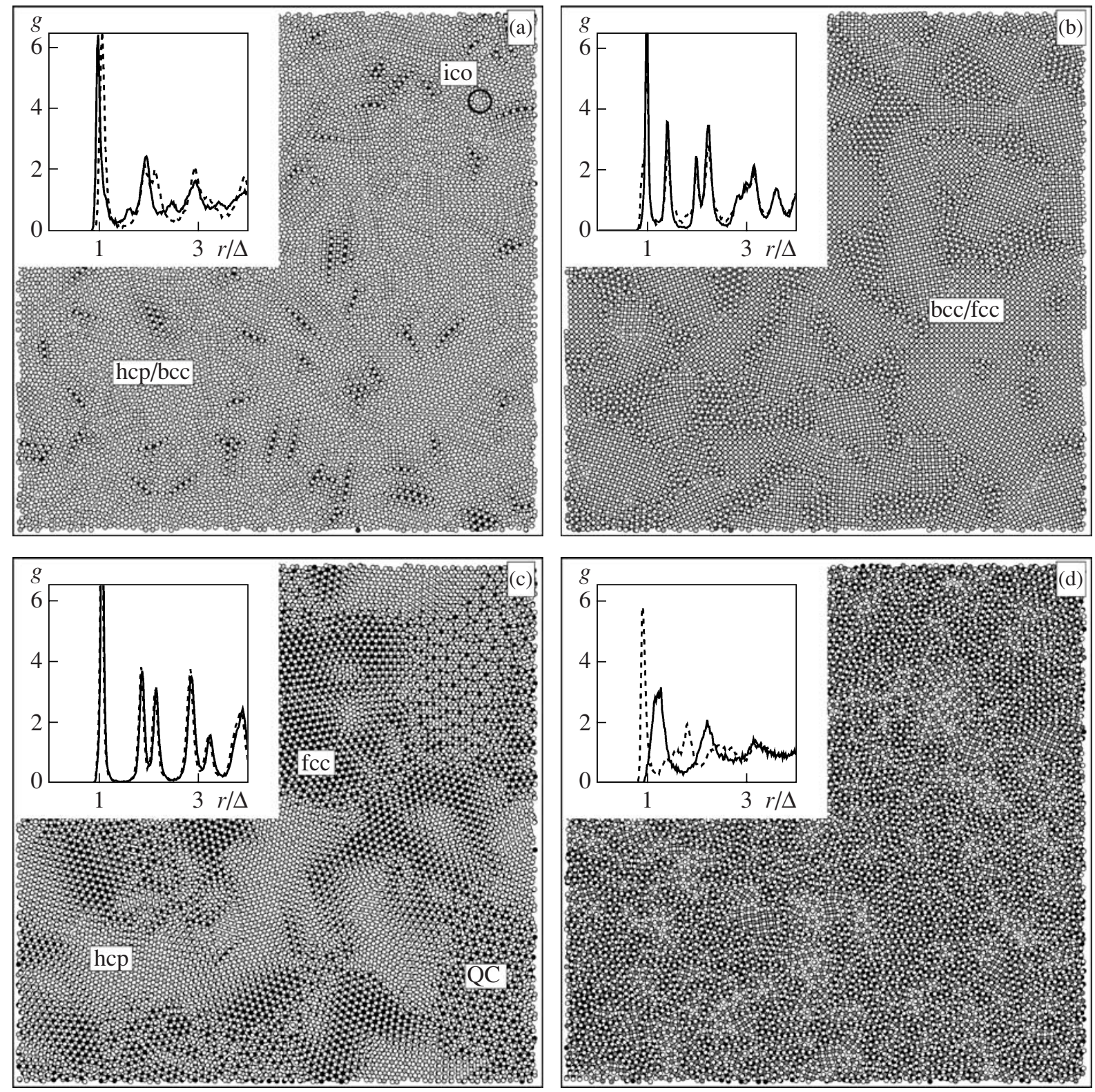

Fig. 3. Dust crystallization in a narrow channel for $(a, b)$ parabolic and (c, d) hard-wall confinement. The grayscale value of a grain image depends on coordinate: white, gray, and black images correspond to the first, second, and third layers, respectively. Shown are $(a, c)$ quasi-steady configurations and $(b, d)$ those near the point of transition to the two-layer state. Domains of different lattice types, including those of quasi-crystalline (QC) phase, are represented. Inserts show the pair correlation functions $g(r / \Delta)$ calculated for the corresponding grain distributions. Solid curves represent the central layer. The pair correlation functions obtained for the upper and lower layers (dashed curves) are almost identical for both types of confinement.

a bifurcation point. For both types of confinement, the central-layer density obviously decreases as the system contracts. In hard-wall confinement, the number of grains in the central layer is smaller than in each layer at the boundary [20]. The difference increases as the system contracts, since grains leave the central layer and the layer becomes much sparser near the point of transition to two-layer structure. As the three-layer system expands and transforms into four layers, the total number of grains in the central two layers after the bifurcation approximately equals that in the stable intermediate regime (see Fig. 2b), because these layers consist of the grains of the single central layer.

In parabolic confinement, the central layer contains more grains than each layer at the boundary and loses them as the system contracts (see Fig. 2a). In this case, a three-layer Yukawa system with equal number of grains in each layer can easily be created, and a perfect plasma crystal can be produced by its crystallization. Figures $3 \mathrm{a}, 3 \mathrm{~b}$ and $3 \mathrm{c}$, 3d illustrate the location of dust grains in a three-layer Yukawa system in parabolic and hard-wall confinements, respectively. Here, the quasi- 
steady crystalline phase of a Yukawa system is shown for $\Gamma \sim 10^{4}$. The grayscale value of a grain image corresponds to a particular layer (coordinate on the $z$ axis). Figures $3 \mathrm{a}$ and $3 \mathrm{c}$ correspond to the intermediate density distributions represented by solid curves in Fig. 2, and Figures $3 \mathrm{~b}$ and $3 \mathrm{~d}$ illustrate the state of the system near the 3-2 transition point for the two confinement types discussed here.

It is clear that parabolic confinement mainly results in the formation of crystallites with $A B A$ ordering of lattice planes (where the first layer is screened by the third one), which is characteristic of hcp and bcc phases (Fig. 3a). A relatively small number of clusters (approximately $1 \%$ ) have icosahedral symmetry, with each particle in the central layer having five nearest neighbors located at the boundary. As this system approaches the 3-2 transition point (Fig. 3b), an hcp lattice transforms into a bcc/fcc one; i.e., a phase transition occurs between solid states.

In the case of a hard-wall confinement (Fig. 3c), distinct regions of $A B A$ ordering (hcp/bcc phase) and $A B C$ ordering (third-layer particles) are clearly seen. A significant portion of the dust is organized into fcc and hcp lattices. It is interesting that a new, quasi-crystalline (QC) phase emerges in hard-wall confinement [20]. As the system contracts (Fig. 3d), both QC and hcp phases disappear, and only fcc/bcc domains remain, with numerous stringlike defects. A more detailed discussion of lattice types and local ordering in the systems under study is presented below. tions

The inserts to Fig. 3 show the pair correlation func-

$$
g(r)=\frac{V}{N^{2}}\left\langle\sum_{i}^{N} \sum_{j \neq i}^{N} \delta\left(\mathbf{r}-\mathbf{r}_{i j}\right)\right\rangle
$$

calculated for each layer in the corresponding particle distribution. Solid curves represent the central layers. The pair correlation functions obtained for the upper and lower layers (dashed curves) are nearly identical for both types of confinement. Strong effect of confinement type on $g(r)$ and significant depletion of the central layer caused by contraction are particularly obvious in the case of hard-wall confinement.

Thus, our simulations of dust behavior in narrow channels lead to an important conclusion: both density of charged dust grains and their distribution between layers (and therefore, distribution of defects) can be manipulated by varying the distance between the system's boundaries (or the shielding parameter $\kappa$ ).

It is frequently required to know the lattice type resulting from crystallization in narrow channels. It can be determined by using local rotational invariants [28]. In this approach, the number $N_{\mathrm{b}}(i)$ of nearest neighbors is determined for each $i$ th particle by analyzing Voronoi polyhedra and Delaunay simplices, which is generally smaller than

$$
N_{\mathrm{m}} \approx \frac{4}{3} \pi r_{\mathrm{m}}^{3} \frac{N}{V}
$$

where $V$ and $N$ are the system's volume and total particle number, respectively, and $r_{\mathrm{m}}$ is the first minimum point of the pair correlation function $g(r)$. The bond vectors $\mathbf{r}_{i j}$ between each $i$ th particle and its nearest neighbors $\left(j=1, N_{\mathrm{b}}\right)$ are then used to calculate the parameter

$$
q_{l m}(i)=\frac{1}{N_{\mathrm{b}}(i)} \sum_{j=1}^{N_{\mathrm{b}}(i)} Y_{l m}\left(\theta_{j}, \phi_{j}\right),
$$

where $Y_{l m}(\theta, \phi)$ is are spherical harmonics, and $\theta$ and $\phi$ are the polar angles of the bond vector $\mathbf{r}_{i j}$. Being dependent on the coordinate system, $q_{l m}(i)$ are poorly suited for determining local orientational order, but they can be used to calculate the second- and third-order rotational invariants

$$
\begin{aligned}
q_{l}(i) & =\left(\frac{4 \pi}{(2 l+1)} \sum_{m=-l}^{m=l}\left|q_{l m}(i)\right|^{2}\right)^{1 / 2}, \\
w_{l}(i) & =\sum_{\substack{m_{1}, m_{2}, m_{3} \\
m_{1}+m_{2}+m_{3}=0}}\left(\begin{array}{ccc}
l & l & l \\
m_{1} & m_{2} & m_{3}
\end{array}\right) \\
& \times q_{l m_{1}}(i) q_{l m_{2}}(i) q_{l m_{3}}(i),
\end{aligned}
$$

where $\left(\begin{array}{ccc}l & l & l \\ m_{1} & m_{2} & m_{3}\end{array}\right)$ are Wigner $3 j$ symbols. Since each lattice type is characterized by a unique set of $q_{l}$ and $m_{l}$, the observed crystalline structure can be identified by comparing the corresponding values of $q_{l}^{\text {id }}$ and $w_{l}^{\text {id }}$ with those for perfect lattices.

Crystalline structure is generally identified by using the second-order invariants $q_{4}$ and $q_{6}$ and the thirdorder invariants $w_{4}$ and $w_{6}$ (e.g., see $[28,29]$ ), which can easily be calculated for fcc/hcp/ico/bcc crystals. For fcc/hcp/ico, $N_{\mathrm{b}}=12$ and the values of these invariants are $q_{4}^{\mathrm{fcc}}=0.1909, q_{6}^{\mathrm{fcc}}=0.5745, w_{4}^{\mathrm{fcc}}=-0.1593$, and $w_{6}^{\text {fcc }}=-0.01316$ for the face-centered cubic lattice; $q_{4}^{\mathrm{hcp}}=0.0972, q_{6}^{\mathrm{hcp}}=0.4847, w_{4}^{\mathrm{hcp}}=0.1341$, and $w_{6}^{\text {hcp }}=-0.01244$ for the hexagonal close-packed lattice; and $q_{4}^{\text {ico }}=0, q_{6}^{\text {ico }}=0.6633, w_{4}^{\text {ico }}=-0.1593$, and $w_{6}^{\text {ico }}=-0.1697$ for the icosahedral lattice.

Since $q_{6}^{\text {cr }}$ is large for all lattice types considered here, it can be used to examine early nucleation/crystallization stages in various systems (e.g., see [29]). For weakly ordered systems, such as gases or liquids, the average $\left\langle q_{6}\right\rangle \approx N_{\mathrm{b}}^{-1 / 2}$ is much smaller than $q_{6}^{\text {cr }}$; for example $\left\langle q_{6}\right\rangle \approx 0.29$ for $N_{\mathrm{b}}=12$. 

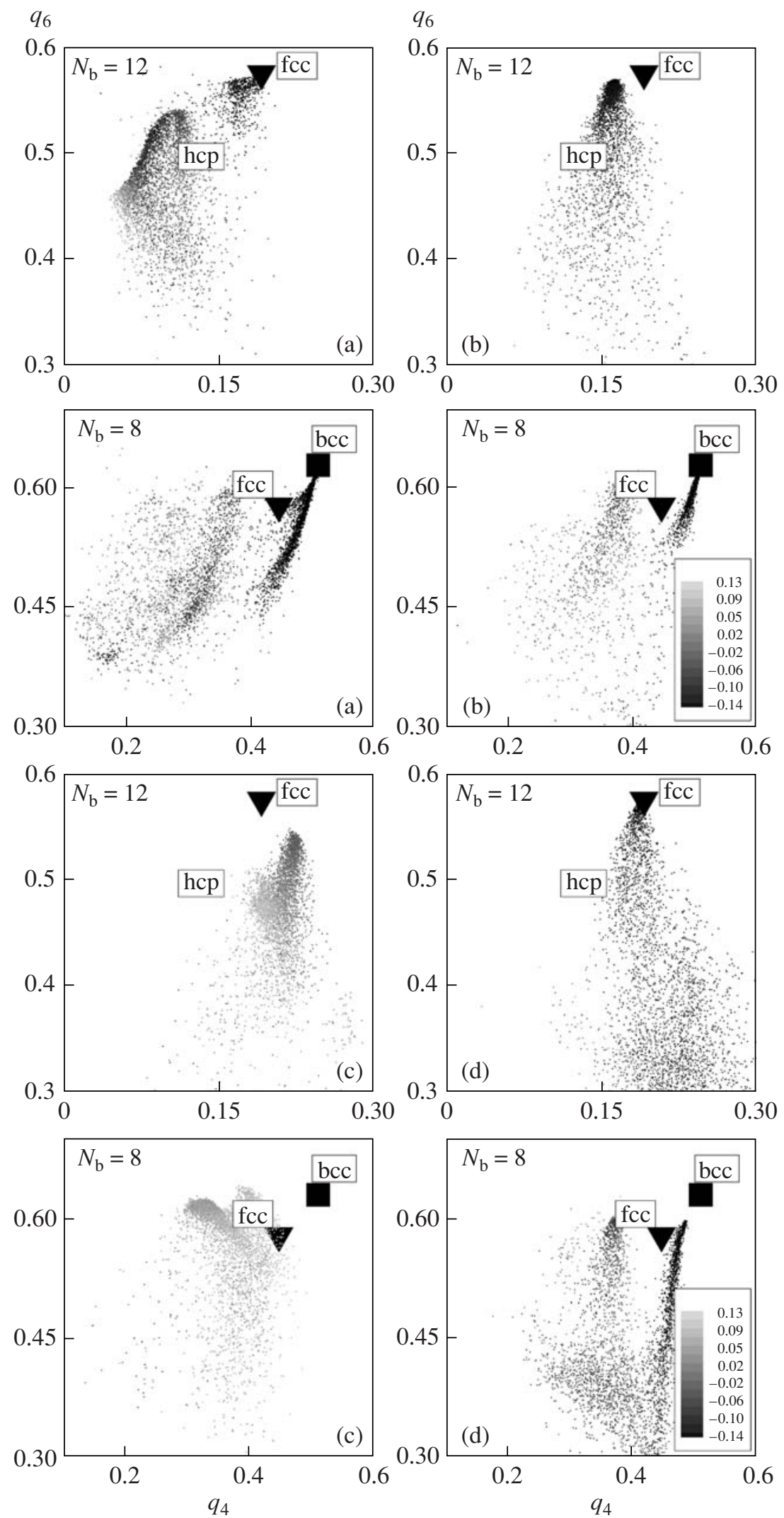

Fig. 4. Local rotational invariants $q_{4}$ and $q_{6}$ of dust grains in the central layer calculated by using data presented in Fig. 3 for (a, b) parabolic and (c, d) hard-wall confinements. The grayscale value corresponds to $w_{4}$. 


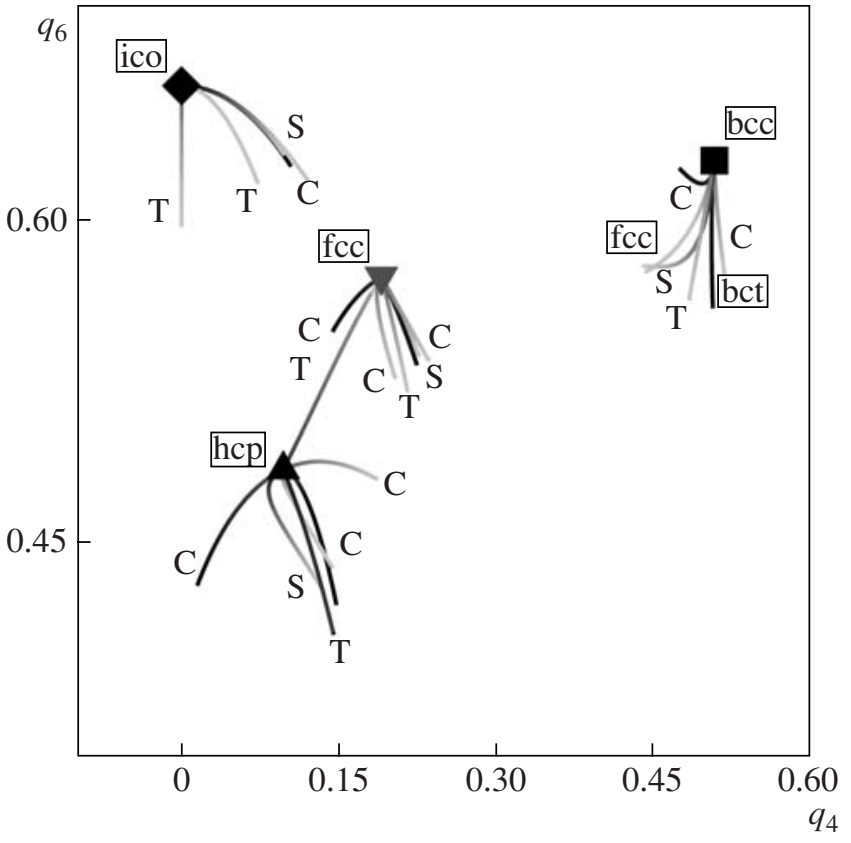

Fig. 5. Variation of local rotational invariants caused by compression/dilation (C), shear (S), and torsion (T) for fcc/hcp/ico and bcc lattices calculated by taking into account 12 and 8 nearest neighbors, respectively. Also shown are rotational invariants for fcc (calculated by using eight nearest neighbors) and bct lattices.

For the simple cubic lattice (with $N_{\mathrm{b}}=8$ ), $q_{4}^{\mathrm{sc}}=$ $0.5092, q_{6}^{\mathrm{sc}}=0.6285, w_{4}^{\mathrm{sc}}=-0.1593$, and $w_{6}^{\mathrm{sc}}=0.1316$. To identify bcc clusters, it is frequently required to take into account the second neighbor shell, which is located at a relatively close distance $((2 / \sqrt{3}-1) \Delta \approx 0.15 \Delta)$ from the first one. The corresponding second-nearestneighbor number is $N_{\mathrm{b}}=14$, while $q_{4}^{\mathrm{bcc}}=0.0363$, $q_{6}^{\mathrm{bcc}}=0.510, w_{4}^{\mathrm{bcc}}=0.1593$, and $w_{6}^{\mathrm{bcc}}=0.01316$. Even though $q_{l}$ and $w_{l}$ for various lattices have been published, in part, in the pioneering study [28], we list them here because incorrect values can frequently be found in the literature. Figure 4 shows the distribution of the local rotational invariants $q_{4}, q_{6}$, and $w_{4}$ for the grain distributions shown in Figs. $3 \mathrm{a}$ and $3 \mathrm{~b}$ for parabolic confinement and in Figs. $3 \mathrm{c}$ and $3 \mathrm{~d}$ for hard-wall confinement. For parabolic confinement, almost perfect hcp/fcc/bcc phases are easily identified in Fig. 3a, and the disappearance of hcp and fcc phases (domination of the bcc phase) resulting from the 3-2 transition is obvious (see Fig. 3). For hard-wall confinement, fcc and hcp phases are easily identified, while the bcc phase is almost absent (Fig. 3c), and small fcc/bcc clusters remain as the 3-2 transition point is approached (see Fig. 3).

The lattice of a real plasma crystal is always distorted because of various factors. For example, a difference in slow particle drift between different regions of the crystal can give rise to shear stresses and, therefore, to its local distortion. Rotational motion can give rise to torsional defects on scales comparable to $\Delta$. Crystalline structure can also be distorted by short-wavelength acoustic disturbances. Anisotropy of the particle interaction potential (due to the ion flux to the walls in the electron region of RF discharge), as well as dipoledipole interaction between grains, may cause lattices of other types to appear in the system. For example, in addition to the bcc lattice (with $a=b=c$ ), bct (bodycentered tetragonal) and bco (body-centered orthorhombic) phases may form (with $a=b \neq c$ and $a \neq b, c \neq$ $a, c \neq b$, respectively). In narrow channels, distorted fcc/hcp clusters may form, depending on the confinement type. Therefore, of interest are the changes in $q_{l}$ and $w_{l}$ due to various distortions of fcc/hcp/ico/bcc lattices. Data of this kind are given in Fig. 5, where the variations of $q_{l}$ and $w_{l}$ due to weak shear, compression/dilation, and torsion of these lattices (without change in the nearest neighbors) are shown on the $q_{4} q_{6}$ plane. Note that these distortions generally reduce the value of $q_{6}$ for all of the lattice types. Note also that local invariants are more sensitive to torsion and less sensitive to shear and compression. The data shown in Fig. 5 can be used to find dilated hcp/fcc lattices and quasi-crystalline phase regions (torsional defects of fcc/hcp lattice) in Fig. 3c.

In summary, molecular dynamics simulations are used in this study to examine the effect of the confining potential on crystallization of Yukawa particles systems in 3D narrow channels. The parabolic and hard-wall confinements considered here as models of "soft" and "hard" confinements induce different behavior of dust density at the boundaries and thus have strong affect local order and lattice type. Compression or dilation of the examined three-layer Yukawa system leads to bifurcation and formation of a two- or four-layer system, respectively. This results in significant changes in dust density and transverse distribution between layers, suggesting that dust-grain flux can easily be manipulated in such systems. This conclusion has is extremely important for applications in nano- and micromechanics and physics of nano- and microliquids.

\section{REFERENCES}

1. P. K. Shukla and A. A. Mamun, Introduction to Dusty Plasmas Physics (Institute of Physics, London, 2002).

2. V. E. Fortov, A. V. Ivlev, S. A. Khrapak, et al., Phys. Rep. 421, 1 (2005).

3. M. Horányi, T. W. Hartquist, O. Havnes, et al., Rev. Geophys. 42, RG4002 (2004).

4. B. A. Klumov, S. V. Vladimirov, and G. E. Morfill, Pis'ma Zh. Éksp. Teor. Fiz. 85 (10), 584 (2007) [JETP Lett. 85 (10), 478 (2007)].

5. B. A. Klumov, S. I. Popel, and R. Bingham, Pis'ma Zh. Éksp. Teor. Fiz. 72 (7), 524 (2000) [JETP Lett. 72 (7), 364 (2000)]. 
6. B. A. Klumov, G. E. Morfill, and S. I. Popel, Zh. Éksp. Teor. Fiz. 127 (1), 171 (2005) [JETP 100 (1), 152 (2005)].

7. B. A. Klumov, S. V. Vladimirov, and G. E. Morfill, Pis'ma Zh. Éksp. Teor. Fiz. 82 (10), 714 (2005) [JETP Lett. 8 (10), 632 (2005)].

8. B. A. Klumov and G. Morfill (2008) (in press).

9. V. E. Fortov, Yu. N. Gnedin, M. F. Ivanov, et al., Usp. Fiz. Nauk 166 (4), 391 (1996) [Phys.-Usp. 39 (4), 363 (1996)].

10. G. Morfill, A. V. Ivlev, S. A. Khrapak, et al., Contrib. Plasma Phys. 44, 450 (2004).

11. G. Morfill, S. A. Khrapak, A. V. Ivlev, et al., Phys. Scr., T 107, 59 (2004).

12. G. Morfill, M. Rubin-Zuzic, H. Rothermel, et al., Phys. Rev. Lett. 92, 175004 (2004).

13. M. Rubin-Zuzic, G. E. Morfill, A. V. Ivlev, et al., Nat. Phys. 2, 181 (2006).

14. B. A. Klumov, M. Rubin-Zuzic, and G. E. Morfill, Pis'ma Zh. Éksp. Teor. Fiz. 84 (10), 636 (2006) [JETP Lett. 84 (10), 542 (2006)].

15. H. K. Christenson, J. Phys.: Condens. Matter 13, R95 (2001).

16. C. Alba-Simionesco, B. Coasne, G. Dosseh, et al., J. Phys.: Condens. Matter 18, R15 (2006).

17. R. D. Deegan, O. Bakajin, T. F. Dupont, et al., Nature 389 (6653), 827 (1997).
18. M. G. Clerk, P. Cordero, J. Dunstan, et al., Nat. Phys. 4 (3), 249 (2008).

19. B. A. Klumov and G. E. Morfill, Pis'ma Zh. Éksp. Teor. Fiz. 85 (10), 604 (2007) [JETP Lett. 85 (10), 498 (2007)].

20. B. A. Klumov and G. E. Morfill, Pis'ma Zh. Éksp. Teor. Fiz. 87 (8), 477 (2008) [JETP Lett. 87 (8), 409 (2008)].

21. M. Schmidt and H. Lowen, Phys. Rev. E: Stat. Phys., Plasmas, Fluids, Relat. Interdiscip. Top. 55, 7228 (1997).

22. A. Fortini and M. Dijkstra, J. Phys: Condens. Matter 18, L371 (2006).

23. J. H. Chu and L. I, Phys. Rev. Lett. 72, 4009 (1994).

24. H. Thomas, G. E. Morfill, V. Demmel, et al., Phys. Rev. Lett. 73, 652 (1994).

25. H. Ikezi, Phys. Fluids 29, 1764 (1986).

26. L. W. Teng, P. S. Tu, and L. I, Phys. Rev. Lett. 90, 245004 (2003).

27. S. Hamaguchi, R. T. Farouki, and D. H. E. Dubin, Phys. Rev. E: Stat. Phys., Plasmas, Fluids, Relat. Interdiscip. Top. 56, 4671 (1997).

28. P. J. Steinhardt, D. R. Nelson, and M. Ronchetti, Phys. Rev. B: Condens. Matter 28, 784 (1983).

29. S. Auer and D. Frenkel, J. Chem. Phys. 120, 3015 (2004).

Translated by A. Betev 